\title{
LA IMPORTANCIA DE LOS PUERTOS DENTRO DE LA ECONOMÍA EN COLOMBIA Y SUS PAISES FRONTERIZOS
}

\author{
Juan David Bobadilla Falla \\ Programa de Administración de Empresas \\ Administrador de Empresas \\ jdbobadilla@poligran.edu.co \\ Allynson Venegas Camargo \\ Programa Administración de Empresas \\ Docente \\ Magíster en docencia \\ vcallynson@poligran.edu.co
}

POLITÉCNICO GRANCOLOMBIANO

ESCUELA DE NEGOCIOS, GESTIÓN Y SOSTENIBILIDAD

ADMINISTRACIÓN DE EMPRESAS

BOGOTÁ - COLOMBIA

2018 


\section{Resumen}

Por contribuir la actividad portuaria al desarrollo económico de las naciones y representar un factor estratégico en el comercio internacional, el presente artículo expone el desarrollo histórico, crecimiento e influencia de la actividad portuaria en el mundo y enfatiza su importancia para el desarrollo económico de países latinoamericanos, especialmente para Colombia frente a las naciones fronterizas, ya Colombia se ubica dentro del ranking de los 20 países con mayor actividad portuaria, lo cual no solo representa un papel esencial en el tráfico exterior, sino que también contribuye al crecimiento de una nación por haberse convertido en centros logísticos de transporte intermodal de primer orden en los que se realizan muchas otras actividades de valor añadido fuera del tráficos comercial como lo son la generación de ingresos para las arcas del estado (tasas portuarias), atención de tripulaciones, la promoción de empleos, así como la gestión de la información que se intercambia entre los diferentes agentes que intervienen en todas estas actividades.

Palabras Claves: puertos marítimos, desarrollo portuario y económico.

\section{Desarrollo Histórico de los Puertos}

El comercio marítimo a través de la historia ha representado un importante papel dentro del desarrollo económico de los países a nivel mundial. Es por ello que la historia nos remonta a la práctica humana de mercadear productos, el famoso trueque ancestral como medio de canje, cuyo hábito milenario ha evolucionado hasta lo que hoy llamamos intercambio comercial. Al respecto Encinas (2017) señala:

El puerto ha sido desde tiempos inmemoriales un centro de comercio estratégico que puso en su momento a los Estados Nación de la antigüedad como Fenicia, Grecia, Egipto, Roma, España, o China, en primer plano. Su importancia fue motivo de disputas entre países, así como de unificación en el movimiento de cargas y estas condiciones se mantienen hasta nuestros días.

Posiblemente los puertos empezaron siendo maravillas de la ingeniería, pero las tareas de carga y descarga seguían haciéndose con la maquinaria más antigua, la fuerza humana. Generalmente esas tareas eran hechas por las personas que habitaban las diferentes costas; de allí que, los investigadores en el área infieren que el desarrollo de la navegación contó, desde el principio, con determinados lugares de la costa (bahía y ensenadas, así como desembocaduras de ríos) que ofrecieron posibilidades de atranque y refugio seguro a las primitivas embarcaciones.

Se conoce que los primeros grandes navegantes del Mediterráneo fueron los cretenses, pero poco ha quedado hoy en día de sus infraestructuras portuarias. Las primeras obras de este tipo de las que ese tiene constancia, 2000 años ante de Cristo, se deben a egipcios y fenicios. Así, las primeras referencias arqueológicas correspondientes a un puerto se han hallado junto a la isla de Pharos, en Egipto, famosa especialmente por la torre iluminada que servía de guía a los barcos y que ha dado nombre genérico a estas construcciones. Este puerto fue construido bajo el reinado del faraón Serunset, destruido y sepultado bajo las aguas posteriormente por un terremoto y reconstruido más tarde por Alejandro Magno. Se dice que los obreros egipcios enterraban la barcaza en la arena de la ribera del río, después utilizaban sogas y trineos para arrastrar los obeliscos o monumentos y colocarlos sobre unas embarcaciones que estaban cubiertas de arena. Después, se quitaba la arena para que pudiera salir la embarcación a flote. De esta manera se embarcaban las cargas extra-dimensionadas.

Los fenicios, griegos, vikingos y polinesios hicieron amplio uso de estos lugares privilegiados, tanto en su comercio y actos de piraterías, como en sus migraciones. Los primeros no solo dotaron de faros a los puertos naturales para favorecer la navegación nocturna, sino que fueron los iniciadores de la construcción de puertos artificiales. Así, en el siglo XIII a.C., los fenicios construyeron puertos de piedra en Tiro y Sidon, con una técnica tan perfecta que aún persiste sus partes fundamentales. 
Si bien la carga y descarga de la época primitiva no era algo resaltante, no puede decirse lo mismo de la construcción de los puertos romanos, ya que eran considerados como las puertas del imperio, época durante la cual se construyeron grandes obras marítimas que no pudieron ser igualadas en los 1500 años posteriores y de las que han quedado numerosos restos, algunos de los cuales siguen en uso incluso hoy en día. Ejemplos de estas construcciones son los puertos de Ostia (Roma), Centum Collae (Civitavechia), Massilia (Marsella), Leptis Magna (Libia), ... De este período han sobrevivido los primeros textos y tratados que versan sobre construcción portuaria, como el de Vitrubio y allí empezó la implementación de los barriles como el principal medio de almacenamiento, ya que era por su forma de rueda facilitaba en parte su movilización. Pero, por más perfecto que fuera, terminaba dependiendo de la mano de obra humana. Los romanos también promovieron la construcción de faros a lo largo de las costas mediterránea y atlántica, incluyendo los de Ostia, Boulogne (Francia), Dover (Reino Unido), o el faro de Hércules (A Coruña), que en la actualidad sigue aún en funcionamiento

En el otro extremo del mundo, en China, también hubo un importante desarrollo de la navegación, aunque no quedan restos de su infraestructura portuaria si la hay de la red de canales que se creó para facilitar el tráfico fluvial, algunos de los cuales siguen en funcionamiento en la actualidad.

A lo largo de la Alta Edad Media, no se producen grandes avances en la construcción portuaria. De hecho, se trata de un largo periodo recesión en lo que respecta al comercio marítimo y a la navegación en general. El imperio Bizantino, heredero del imperio Romano, mantiene las infraestructuras en su zona de influencia, pero no realiza nuevas grandes obras.

Los musulmanes, por su parte, nunca mostraron un gran interés por desarrollar una flota de importancia; al respecto solo cabe destacar que el califato de Córdoba estableció para el Mediterráneo un sistema portuario basado en tres ciudades arsenales; Almería, Denia y Tortosa, que tuvo dos siglos de vigencia.

A partir de los siglos X y XI comienza a producirse un resurgimiento del comercio en el Mediterráneo encabezado por las nacientes repúblicas italianas (Pisa, Venecia, Génova), los territorios marítimos del sur de Francia y, especialmente, la corona Catalanoaragonesa.

A finales del siglo XII y como elemento básico de la alasocracia catalano-aragonesa aparecen los Consulados, instituciones jurídicas cuya finalidad es la de resolver litigios entre comerciantes pero cuya actividad se extendió a todos los campos del comercio y la navegación. A partir del siglo XIII comienzan a aparecer una serie de ordenanzas y reglamentos para regular las actividades marítimas y comerciales que culminan con la redacción del Llibre del Consolat del Mar, que fue la base de la jurisprudencia marítima europea y cuya vigencia se mantuvo hasta 1829, año de la promulgación del Código de Comercio.

Paulatinamente, los puertos fueron acogidos en una población diversificada (cargadores o estibadores, comerciantes, etc.) e incorporando nuevas instalaciones adecuadas a las necesidades impuestos por el creciente tráfico marítimo que hicieron de ellos complejas unidades técnicos - comerciales, como ocurrió en la edad media europea con los puertos del mar del Norte, confederados en la liga Anseática o en los del mar Mediterráneo (Génova, Venecia y Barcelona)

En 1492, con el descubrimiento del Nuevo Mundo, el foco de la actividad marítima y comercial que hasta ahora se centraba en el Mediterráneo se gira hacia el Atlántico. El interés en establecer un control de la Administración sobre las operaciones comerciales con las Indias y con el fin de proteger los buques de los ataques de piratas y corsarios (para lo cual se estableció la navegación en régimen de flotas) hizo que la Corona otorgase a determinados enclaves portuarios estatutos de privilegio que permitieron su desarrollo. Es el caso de la concesión del monopolio por parte de los Reyes Católicos del comercio con América al puerto de Sevilla, que en 1711 pasaría al de Cádiz. Ello genera la decadencia de los puertos de Mediterráneo. La exclusividad en el tráfico con América dura hasta 1778, año en que el rey Carlos III liberaliza las relaciones comerciales con el Nuevo Mundo. 
Durante el reinado de Carlos IV y promovidas por el Marqués de la Ensenada, se dictan las Ordenanzas Generales de la Armada que establecen los principios de una política general de puertos. Este primer intento serio de organizar un sistema portuario se basó en la construcción de tres grandes arsenales en El Ferrol, La Carraca y Cartagena. A pesar que desde finales del siglo XVII se fueron llevando a cabo intentos de aplicar el vapor a la navegación no es hasta 1807 que Fulton, recogiendo todas las experiencias anteriores, pune en servicio el primer barco de vapor completo, el Clermont, que realizó el servicio entre Nueva York y Albany sobre el río Hudson. Al siguiente año el norteamericano Stevens construyó un barco de vapor que realizó la primera travesía marítima mediante este sistema.

A comienzos del siglo XIX, la utilización masiva del vapor en los buques, en lugar del viento, permitió aumentar notablemente su tonelaje y capacidad de carga y como consecuencia, tecnificar más todas las inhalaciones portuarias; ya no se necesitaban diez hombres para mover una carga sino simplemente una caldera y un motor a vapor que podía disminuir tiempos a un menor costo.

En 1956, con el transporte de 58 contenedores de Port Newark a Houston a bordo del Ideal $\mathrm{X}$, se pone en funcionamiento el primer transporte marítimo de mercancía contenerizada, dando vida a una idea que Malcolm McLean tuvo 20 años antes.

La aparición del contenedor causó una auténtica revolución en el diseño de los puertos cambiando enormemente su aspecto físico. En efecto, el empleo de contenedores, junto con el uso de equipamiento especial para su manipulación hace que los procesos de carga y descarga de un buque sean mucho más rápidos que en el caso de cargas no contenerizadas. Esta rapidez en la operativa hace que no sea necesaria tanta línea de muelle, puesto que el tiempo de estancia de los buques es mucho menor, pero en cambio se precisen grandes explanadas para el depósito de contendores. Comparando los diseños de los puertos antes y después de la aparición del contendedor podemos observar las implicaciones que este ha tenido en la construcción portuaria. Los buques no debían estar días enteros o semanas en los puertos, ya que los barcos podían realizar trayectos de dos semanas y cargar o descargar mercancías en solo quince minutos obteniendo una reducción considerable en los costos.

En 1880 aparece la primera Ley de Puertos, en la que ya se hace una distinción entre los puertos de interés general (que se clasifican en puertos de primer y segundo orden, manteniendo el número de 13) y puertos de interés local. Así mismo reconoce el dominio público del litoral y la gestión del conjunto del sistema dependiente de la administración central. En 1869 se inaugura el canal de Suez; en 1914 lo hace el canal de Panamá. Como consecuencia de la construcción de estas importantes infraestructuras se producen cambios en las principales rutas de navegación, puesto que evitan la necesidad de rodear África y América del Sur para alcanzar Europa desde el Índico y el Pacífico respectivamente. (Rúa, 2006, p. 4-6).

Todos estos cambios a través del tiempo han ido mejorando de la mano con la tecnología, los procesos logísticos en los puertos del mundo:

Si bien atribuimos el avance de la Globalización a las circunstancias políticas y macroeconómicas de la segunda parte del siglo XX, no debemos olvidar que el bajo coste y la eficiencia del transporte marítimo han sido esenciales para este desarrollo. (...) Dada la complejidad del negocio de transporte marítimo y su importancia para las economías locales y nacionales, es imprescindible aplicar los métodos más avanzados para aprovechar las grandes oportunidades económicas que existen en el sector de transportes marítimos (Álvarez, 2006).

En la actualidad, los puertos marítimos permiten el transporte de un gigantesco volumen de mercaderías por todo el mundo, ocupando un papel prioritario dentro de la demanda comercial internacional y el desarrollo de la competencia entre sus diferentes terminales de carga.

\section{Desarrollo Portuario y Económico}

La actividad portuaria representa una alternativa real para el crecimiento económico y comercial de las naciones, pues son fundamentales para el intercambio comercial internacional por ser lugares de resguardo de las 
embarcaciones en las que se desarrollan operaciones de carga, descarga, embarque y desembarque de gran cantidad de mercancías necesarias para el consumo de las poblaciones. En este sentido, Encinas (2017), expone: "El transporte marítimo es actualmente el medio más eficaz de transportar mercancías, su bajo costo, gran capacidad de transporte masivo de mercancías, movimientos en largas distancias son algunas razones que sostienen este mecanismo comercial como el más conveniente y rentable".

Por ser los puertos parte de la cadena de transporte internacional y del comercio mundial en el año 2001, la Comisión Económica para América Latina y el Caribe (CEPAL), expresó que, con el crecimiento del comercio mundial y el movimiento de contenedores, se genera la necesidad de crear buques con mayor capacidad y así disminuir los costos. El crecimiento que ha tenido el movimiento de contenedores en el mundo, para el año 1980, fue de 13.5 millones de TEU's (acrónimo del término en inglés Twenty-foot Equivalent Unit, que significa Unidad Equivalente a Veinte Pies), mientras que para el año 1990 ya se movían 28.5 millones TEU's, en esta década se duplicó el movimiento de contenedores. Para el año 2000, se llegó a los 66 millones TEU's. Junto a esto se observa que el crecimiento es más en valor que en volumen y esto se le puede atribuir al aumento de la tecnología lo que nos permite tener aparatos más pequeños como es el ejemplo de los televisores y los teléfonos celulares que cada día el tamaño disminuye. Este crecimiento del movimiento de mercancías va al mismo tiempo con el crecimiento económico de los países, aunque para el crecimiento de las economías influyen muchos factores, la parte del comercio exterior y la influencia de los puertos es muy significativa. Al respecto, Rúa (2006), explica:

En las últimas décadas se ha producido una intensificación de la competencia entre puertos y una especialización de las operaciones, de tal manera que la unidad operativa básica no es ya el puerto en su conjunto sino las terminales de carga, ubicadas dentro de la zona de servicios portuaria y especializadas en un determinado tipo de tráfico (p. 2).

En este mismo orden de ideas González (2013), expone:

La velocidad con que el transporte marítimo se ha desarrollado en los últimos años ha puesto en serios problemas las capacidades logísticas y de servicios portuarios alrededor del mundo. Un factor determinante es el crecimiento en el tonelaje de los buques mercantes, que les da mayores capacidades de carga y transporte, en particular los portacontenedores; en la actualidad aproximadamente el 93\% de la carga mundial que no es a granel se realiza en contenedores (p. 1).

Ambos autores concuerdan en que debido al avance tecnológico el desarrollo de la globalización económica y el crecimiento de los flujos comerciales, la actividad portuaria influye directamente en crecimiento económico de los países, pues se podría afirmar que la columna vertebral del comercio intercontinental para el transporte a granel de materias primas y la importación y exportación de alimentos y bienes manufacturados.

Por otra parte, una nueva edición de la CEPAL en la cual publica el ranking de movimiento portuario de contenedores según el Perfil Marítimo y Logístico, en el 2016 hubo una agudización general de la desaceleración del comercio exterior en los terminales de contenedores, que es arrastrada por el movimiento portuario desde hace varios años: 6,0\% en 2012, 1,3\% en 2013, 2,4\% en 2014 y 2,5\% en 2015. El deterioro en 2016 estuvo determinado principalmente por las disminuciones en la actividad de cinco países: Brasil $(-4,4 \%)$, Panamá $(-9,1 \%)$, Colombia $(-3,6 \%)$, Argentina $(-6,1 \%)$ y Bahamas $(-14,3)$.

En este informe Colombia se encuentra en el tercer lugar de actividad portuaria marítima, pero con un porcentaje bajo con respecto a Panamá y a Brasil como principales puertos de Latinoamérica.

Ahora bien, la CEPAL muestra un informe anual en el que muestra el ranking detalladamente de los movimientos de carga en contenedores en 120 puertos de la región, con base a información recopilada directamente con las autoridades portuarias y operadores de los terminales marítimos por parte de la misma entidad y en el informe del 2016, expone que esta actividad disminuyó $0,9 \%$, con un volumen aproximado total de 47,5 millones de TEU, mostrando así los puertos ubicados en los primeros 20 lugares del ranking. A continuación, se presenta el gráfico. 


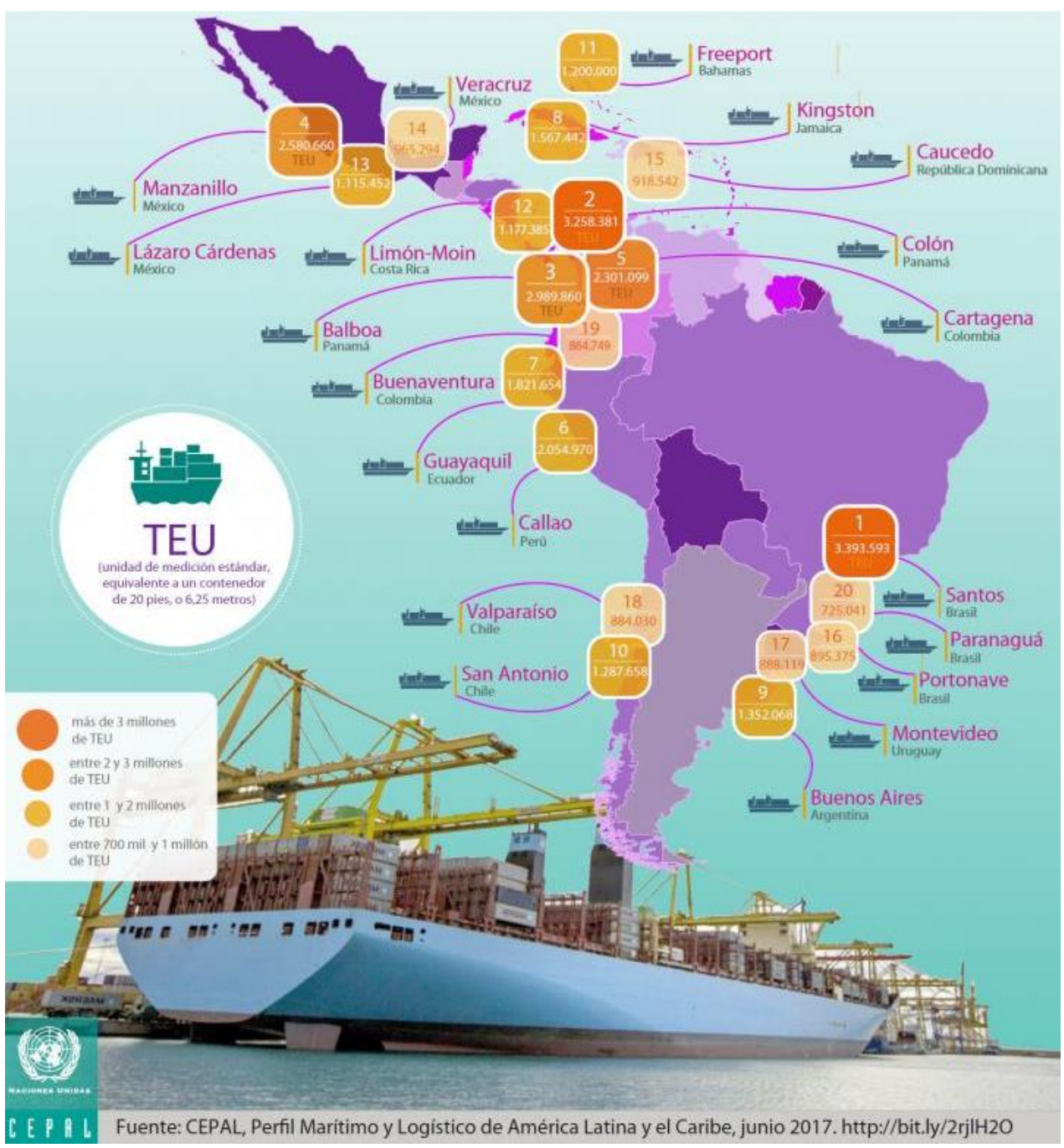

Fuente: Cepal (2017)

En la imagen se puede apreciar que de los veinte lugares (20), los tres primeros son ocupados por los Puertos de: Santos (Brasil), Colón y Balboa (Panamá); así mismo, el puerto de Cartagena y Buenaventura (Colombia) se encuentran dentro de ranking, de quinto (5to) y de décimo novena lugar (19no), lo que indica que las actividades portuarias representan para Colombia un importante desarrollo económico, frente a sus vecinos latinoamericanos como Panamá, Brasil y Perú, que destacan por el desarrollo de actividades económicas importantes a nivel mundial, especialmente Panamá debido a su Canal.

Se debe destacar que, el puerto del Callao en Perú también destaca dentro de los 20 países ocupando el sexto lugar (6to), más Ecuador no se encuentra dentro del ranking.

\section{Tabla 1. Ranking de Puertos Latinoamericanos}

\begin{tabular}{ll}
\hline Puerto Latinoamericano & TEU $^{1}$ \\
& \\
\hline Santos, Brasil & 3.391 .593 \\
\hline
\end{tabular}

${ }^{1}$ TEU. Unidad de medición estándar, equivalente a un contenedor de 20 pies o 6,25 metros. 


\begin{tabular}{ll}
\hline Puerto Latinoamericano & TEU \\
& \\
\hline Colón, Panamá & 3.258 .381 \\
Balboa, Panamá & 2.989 .860 \\
Cartagena, Colombia & 2.301 .099 \\
Buenaventura, Colombia & 1.821 .654 \\
Callao, Perú & 2.054 .970 \\
\hline
\end{tabular}

Fuente: CEPAL (2017), Perfil Marítimo y Logístico de América Latina y el Caribe 2016

Tabla 2: Ranking Latinoamericano de Países

\begin{tabular}{ll}
\hline Puerto País & TEU \\
\hline Puertos de Panamá & 6.248 .243 \\
Puertos de Brasil & 5.014 .009 \\
Puertos de México & 4.659 .406 \\
Puertos de Colombia & 3.165 .848 \\
Puertos de Perú & 2.054 .970 \\
\hline
\end{tabular}

Fuente: CEPAL (2017) Estudio Económico de América Latina y el Caribe.

Cabe resaltar que, según la publicación Alphaliner a nivel global, durante el 2016 el tráfico de contenedores en puertos también tuvo un bajo dinamismo, sus estimaciones arrojan que los volúmenes en los 100 primeros puertos de contenedores del mundo se elevaron tan solo un 1,8\% en 2016, a 555,6 millones de TEU

\section{Puertos Latinoamericanos/Suramericanos}

En Latinoamérica existen una gran cantidad de puertos, los cuales representan una de las principales vías de comunicación y comercialización para cada uno de sus países. El desarrollo de los mismos ha evolucionado según las necesidades propias de la región y más aún de los sistemas de producción de sus países. Entre los principales puertos se pueden mencionar:

\subsection{Brasil: Puerto de Santos}

Es el principal puerto de Brasil y de América Latina. Se encuentra localizado en la ciudad de Santos, en el Estado de São Paulo. El sistema de accesos terrestres al puerto está conformado por las autopistas Anchieta e Imigrantes y por dos líneas de ferrocarril (Ferroban y MRS). Tiene un área de 7.770.000 metros cuadrados.

La ciudad de Santos se localiza en el punto más adecuado para poder trasladar al exterior las mercancías de la zona. La estructura ferroviaria, iniciada en el período del Imperio, garantizó el aflujo de cargas, principalmente café, destinadas al comercio exterior.

Ubicado a 70 kilómetros de la ciudad más grande de América del Sur, São Paulo, el puerto es la salida al exterior de los principales distritos industriales del Gran São Paulo y del complejo industrial de Cubatão. 
Según datos de la Comisión Económica para América Latina y el Caribe, para el año 2014, más del 17\% del tráfico de contenedores en América Latina fue movilizado en Brasil, seguido por Panamá (15\%), México (10.7\%), Chile (7.9\%) y Colombia (7.4\%) (Comisión Económica para América Latina y El Caribe, 2014).

El puerto de Santos según cifras oficiales representa el 67\% del producto interno bruto (PIB) de Brasil (Autoridad Portuaria de Porto de Santos, s/f), lo cual deja claro su amplia influencia comercial en Brasil, y lo que esto representa para la región.

\subsection{Panamá: Puerto Balboa y Puerto Colón.}

\subsubsection{Puerto Balboa.}

Con un total de 30 hectáreas dedicadas al almacenamiento de contenedores y 5 muelles para barcos portacontenedores, Balboa opera con 25 grúas pórticas (10 Post Panamax, 8 Panamax y 7 Super Post Panamax) y 47 RTGs. Las operaciones de trasbordo de carga contenerizada representan el $92.8 \%$ del total de movimiento de contenedores, mientras que el resto está dirigido al mercado local. Un acceso directo con el ferrocarril permite el trasbordo de contenedores con destino a los puertos de Colón. Balboa también tiene capacidad de recibir y despachar graneles secos y líquidos, así como carga especializada, disponiendo de un total de 3,612 conexiones para refrigerados.

En el 2014, este puerto tuvo un movimiento total de 3.2 millones de TEUs, en el que el transbordo representó el 92.3\% del total de TEUs manipulados. En 2015, disminuyó sus movimientos a 3.1 millones de TEUs y así mismo, la proporción de movimientos de trasbordo disminuyó a $90.5 \%$ de los movimientos totales. En el 2016, disminuyó sus movimientos a 2.8 millones de TEUs con un total de $89.6 \%$ de trasbordo. (Georgia Tech Panama Logistics Innovation and Research Center, 2017).

\subsubsection{Puerto Colón.}

Colón Container Terminal, está localizada en Colón Solo Norte, provincia de Colón. Este puerto se está convirtiendo en un punto ideal para trasbordo y carga entrando y saliendo hacia y desde la Zona Libre de Colón. Desde la entrada Atlántica del Canal de Panamá, CCT da servicio a los mercados regionales del Caribe, América del Norte, Central y Sur con embarques originados principalmente en el Lejano Oriente.

Esta terminal posee un área total de 74.33 hectáreas, un canal de acceso de 16.4 metros de calado y una dársena de maniobras con un radio de 600 metros para la rotación de naves. El acceso de entrada del rompeolas posee 200 metros convirtiéndose en una vía dedicada para la Bahía de Manzanillo.

Este puerto posee acceso terrestre hacia la Zona Libre de Colón y al ferrocarril. Cuatro muelles de contenedores, trece grúas pórticas y otros equipos de patio le otorgan a CCT una capacidad de manejo de 2.4 millones de TEUs. Los planes de expansión incluyen la adición de áreas de almacenaje de contenedores a un costado del puerto y cruzando la avenida Randolph, así como la incorporación de más equipos para un manejo más eficiente de las operaciones de contenedores.

Colon Container Terminal manejó 502,706 TEUs en el 2014, en donde el 64.2\% del movimiento total fue transbordo. En el 2015, el movimiento de TEUs aumentó un 57.1\% llegando a manejar 789,663 TEUs en donde el trasbordo representó el 77.6\% del total de movimientos. En el 2016, se registró una disminución del 19.9\% al manejar 632,845 TEUs, donde el trasbordo representó el 75.9\% (Georgia Tech Panama Logistics Innovation and Research Center, 2017). 


\subsection{Colombia: Puerto de Buenaventura y Cartagena}

\subsubsection{Puerto de Buenaventura.}

El primer muelle de Buenaventura fue construido durante la administración del Gobernador del Valle del Cauca, Ignacio Rengifo, y fue administrado por el Departamento hasta 1933 (Henao y Henao, 2009).

Es el puerto más importante del Pacífico, debido al volumen de carga manejada, recientemente así como los que se encuentran en proceso de formalización.

Está constituido por un terminal marítimo que es administrado por la Sociedad Portuaria Regional de Buenaventura S.A. (SPRBUN), y un muelle que es administrado por el Grupo Portuario S.A.; entre los dos reciben cerca del $52 \%$ de la carga que ingresa al país. El gobierno lo dio en concesión a la Sociedad Portuaria Regional de Buenaventura y cuenta con una infraestructura especializada para el manejo de contenedores, granos, carga a granel y de usos múltiples. Tiene 14 muelles, 12 operados por la Sociedad de Buenaventura y 2 bajo otra concesión.

El puerto de Buenaventura es de transporte multimodal. El canal de acceso tiene una longitud de 31,5 kilómetros, equivalente a 17 millas náuticas. Fuera de la bahía: la profundidad es de 9,1 metros en marea baja (Zero Igualdad); dentro de la bodega: la profundidad es de 12 metros en marea baja (igual a cero). Muelle tiene una longitud de 2.001 metros. La profundidad de $12 \mathrm{Mt}$, (en promedio mínimo mareas) limita la llegada de los barcos que requieren más profundidad y limita la carga en peso de los buques.

El puerto de Buenaventura tiene una terminal especializada de contenedores (Buenaventura SA TECSA). Este terminal proporciona servicios con seguridad en la operación, garantizando la disponibilidad y optimización de espacio de la terminal, garantizando la adecuada atención de los clientes.

Dentro de su plan de modernización, SPRBUN -cuyo contrato de concesión otorgado en 1994 fue extendido por 20 años más hasta el 2034- contempla inversiones por 450 millones de dólares, de los cuales 175 millones serán destinados a equipos, 215 millones en infraestructura y 60 millones de dólares en logística.

"Al 31 de diciembre de 2012 habíamos invertido 252 millones de dólares de esos US\$ 450 millones, es decir, que sin haber terminado la primera concesión ya habíamos invertido más del $50 \%$ de las inversiones acordadas con el Estado", comenta Jorge Andrés Gallegos, gerente comercial de la Sociedad.

Recientemente la Sociedad inauguró obras y equipos que aumentarán la capacidad del puerto, de cara a la ampliación del Canal de Panamá. Estas inversiones se traducirán en menores tiempos de operación de los buques en puerto, debido a una atención de cargue y descargue más ágil, que a su vez permitirá importantes ahorros al reducir las tarifas de fl etes para exportadores e importadores.

Según el gerente comercial de la SPRBUN, el año pasado se movilizaron 9.506 .000 toneladas de carga. En cuanto a importaciones, se registraron 7.233.000 toneladas de granel sólido (cereales, fertilizantes y minerales); mientras que las exportaciones sumaron 1.734 .000 toneladas de carga.

De acuerdo con el directivo, los productos que más se reciben a través del puerto son cereales, carga en contenedores, electrodomésticos y el año pasado por dicha terminal ingresaron al país 120 mil vehículos. Lo que más se exporta es azúcar (en contenedores, como carga general y a granel), café y cargas no tradicionales. Este puerto estratégico por su ubicación geográfica, se encuentra dentro del ranking de los 20 países latinoamericanos más importantes por su actividad comercial debido al manejo de los contenedores que son publicados anualmente por la CEPAL, logro importante para Colombia dado que de esta forma se evidencia una gran apertura del desarrollo comercial y económico.

\subsubsection{Puerto de Cartagena.}


Después de cuatro siglos y medio de historia y de ser considerado el puerto de América, la zona portuaria de Cartagena hoy se caracteriza por ser la más grande del país al poseer 54 muelles, dentro de los cuales 18 adelantan actividades de comercio exterior.

De acuerdo con Pérez (2017), este puerto es administrado por la Sociedad Portuaria Regional de Cartagena S.A. (SPRC) desde 1993, es considerado como un auténtico centro logístico integrado que une al Caribe con el resto del mundo, a través de conexiones con más de 288 puertos en 80 países y servicios con las principales navieras del planeta. Dentro de las ventajas que ofrece su infraestructura están sus ocho puntos de atraque con una profundidad de hasta 44 pies (13,41 metros), obras civiles como dragados, construcción de patios y la adquisición de tecnología portuaria de punta.

La transformación tecnológica en el puerto incluye sistemas de información, grúas para apilamiento de contenedores orientadas por satélite y un sistema computarizado para manejo de documentación interconectado con la Aduana, que agiliza la entrada y el despacho de carga, aumentando la seguridad de las operaciones.

Este puerto especializado en el manejo de contenedores alcanzó la cifra récord de 2.018.389 contenedores movilizados al cierre del 2012; logro inédito en Colombia y que lo consolida como el cuarto puerto más importante de Latinoamérica en movilización de carga, después de Balboa y Colón, en Panamá; y Santos, en Brasil.

De acuerdo con la información suministrada por la Sociedad Portuaria, dentro de su plan de modernización "se contemplan inversiones cercanas a los 900 millones de dólares hasta 2017, de los cuales se han ejecutado US\$ 410 millones". Si bien es cierto que no se cumplieron las cifras esperadas, hoy el puerto de Cartagena moviliza 3.000 buques anuales; es decir, el doble que se movilizaba en el año 2012 cuando se realizó la proyección a raíz de la ampliación del canal de Panamá.

Para haber logrado este aumento, la Sociedad portuaria Regional de Cartagena SPRC tuvo que incrementar su despliegue logístico; es decir, que se aumentaron las grúas pórtico a 8 con alcance de hasta 22 contenedores, se aumentó el área de patio a 25 hectáreas con más de 30.000 celdas de almacenamiento, la capacidad anual aumento a 2 millones de TEU y atiende buques de hasta 16.000 TEU y por último se aumentó la profundidad de la dársena de 14 metros a 16.5 metros.

Así mismo, el crecimiento en la otra terminal marítima Conectar ayudó notablemente a aumentar la capacidad anual de 1.5 millones de TEUs a 3.2 millones de TEUs, aumentando las grúas en el muelle de 6 grúas pórtico STS a tener 13 de las mismas, el área de patio paso de ser de 20 hectáreas a 90 hectáreas con más d 58.000 celdas de almacenamiento, de tener 24 grúas RTGs se llegó a una totalidad de 60 grúas RTGs eléctricas que operan de forma silenciosa sin emitir CO2. Las tomas para refrigerados pasaron de 800 a 2.400 tomas equipadas con sensores que monitorean temperatura. De tener 73 tractocamiones, Conectar alcanzó en su flota 120 tractocamiones para el transporte interno de carga. Las bodegas para almacenaje se cuadruplicaron; es decir, de operar con 20.000 metros cuadrados se pasó a operar con 80.000 . Y por último en la puerta de entrada a la terminal marítima, se ampliaron lo carriles de 6 a 12 carriles equipados con tecnología OCR.

"Más allá de sus operaciones portuarias, la Organización Puerto de Cartagena se ha consolidado como una plataforma logística para el comercio global. Su innovador concepto de Centros de Distribución Internacional, $\mathrm{CDI}$, cuenta con un modelo único que se adapta, con un portafolio de servicios, a las necesidades de cada uno de sus clientes. Bajo este concepto compañías de renombre internacional como Samsung, Ford, Pirelli, Bayer, RedBull, DirecTv, entre otras, han encontrado en La Organización Puerto de Cartagena un aliado estratégico para el desarrollo de sus líneas de negocio." (Servicios logísticos y centros de distribución, 2017).

Aunque el crecimiento económico en la región no es el mejor de los últimos años, el Puerto de Cartagena cuenta con toda la infraestructura necesaria para llegar a ese top 3 en el ranking de los puertos latinoamericanos y sin duda, para los próximos años se espera una mejora frente a su actual puesto en el ranking.

\subsection{Perú: Puerto del Callao.}

El callao, considerado como el puerto más importante de Perú está ubicado a solo unos minutos de distancia de la ciudad de Lima por el extremo oeste. . No gozo siempre de la denominación de puerto, ya que en el año 1535 era conocido como puerto de Lima, luego paso a ser llamado como puerto de la ciudad de los reyes. Luego fue callao de la mar. En 1586 finalmente es llamado como el día de hoy es conocido, Puerto del Callao. Hasta el año 1965 se inició la primera construcción formal para empezar a darle forma al puerto que todos en el callao querían, bajo la dirección del ingeniero A. Prentice, con un ancho de 20,5 metros y una longitud total de 
170 metros. En 1868 Templeman Bergmann y Cia presentaron una propuesta para una ampliación considerable del puerto, este proyecto fue aprobado el 16 de agosto de 1869 y la obra fue entregada a finales del año 1877.

Finalmente después de varias modificaciones y ampliaciones desde su inicio, fue aprobado un proyecto presentado por la firma The Frederick Snare Corporation y fue entregado al servicio en octubre de 1934. Fue una estructura impresionante, sin embargo 50 años después alcanzó su límite de vida. En el año 2011 APM Terminals se adjudicó la concesión de la terminal norte multipropósito del Puerto del Callao llevando a cabo los más notorios avances basándose en la experiencia de algunos otros puertos en el mundo.

El puerto del callao ha venido respondiendo al crecimiento económico global, el cual en los últimos años ha generado una inversión en sus instalaciones cercana a los US\$625 millones y todo lo referente a lo estructural; pero, más allá de todo eso, hoy se especula que su crecimiento pueda estar suspendido en medio de la falta de áreas en su entorno. "El puerto del Callao, el aeropuerto Internacional Jorge Chávez y las instalaciones ferroviarias del ferrocarril Central Andino no disponen de espacio suficiente en su interior, y su ampliación a dimensiones óptimas no es posible por la proximidad de la ciudad y el propio desarrollo urbano.”. (Asociación Peruana de Agentes Marítimos, 2017)

La preocupación de la región y del propio país en cuestión pasa por su buen momento económico y el impacto que pueda generar más adelante el puerto del Callao en este. "El valor de las exportaciones (US\$ 2.942,9 millones) aumento en $7,9 \%$, por las mayores ventas de productos tradicionales $(10,7 \%)$ y no tradicionales $(0,9 \%)$ ". (INEI, 2017)

"Dado el crecimiento del comercio exterior en los últimos años, que en más del $80 \%$ se mueve por el puerto del Callao, los espacios en torno a este terminal que se necesitan para el almacenamiento, procesamiento y movilización de la carga de importación y exportación cada vez son más escasas." (Asociación Peruana de Agentes Marítimos, 2017)

La última concesión que adjudico el puerto fue por 30 años en el año 2011 a APM Terminals Callao S.A. con una inversión presupuestada o pactada en 748 millones de dólares destinados al terminal norte multipropósito. Por otra parte, el terminal sur fue otorgado a DP Word Callao desde el año 2006 y con una inversión presupuestada o pactada por 117 millones de dólares. El plazo para llevar a cabo dicha inversión es también de 30 años, al igual que la otra concesión.

Pese a este compromiso que hoy se encuentra pactado, hoy hay incertidumbre por el déficit en áreas para un buen funcionamiento de toda la cadena logística. ¿Aun será para usted una incógnita, si el puerto del Callao pueda seguir avanzando en su crecimiento y logre entrar en ese top tres del ranking mundial de puertos de América Latina?

"Los puertos son empresas que forman parte del sistema integrado de la cadena logística global e interfaces entre los distintos modos de transporte que promueven el desarrollo y la competitividad de los países. Donde las mercancías no solamente están en tránsito sino que también son manipuladas y distribuidas, y en algunos puertos son manufacturadas". (Superintendencia de Puertos y Transporte, 2010)

\subsection{Ecuador: Puerto de Guayaquil}

Guayaquil es el puerto principal de la República del Ecuador, a través del cual se moviliza el 70\% del comercio exterior que maneja el Sistema Portuario Nacional. Fue construido durante el período 1.959 - 1963. Posee una infraestructura adecuada para el desarrollo del comercio internacional, para lo cual cuenta con medios óptimos para la ejecución de las operaciones. En el puerto se prestan todos los servicios requeridos por las naves y las mercaderías a través de operadores privados de alta especialización que, bajo la supervisión de la Autoridad Portuaria, actúan en libre competencia para satisfacer los requerimientos de los usuarios más exigentes, logrando alta eficiencia y reducción de costos. (Autoridad Portuaria de Guayaquil, s/f)

Para el año 2015, según cifras de la Autoridad Portuaria de Guayaquil en materia de importaciones se contabilizaron 5.214.770 de toneladas métricas, lo cual representa el 32\% de las importaciones de ese país y 10.331.050 de toneladas métricas que representan el 15\% de las exportaciones ecuatorianas (Ministerio de Transporte y Obras Públicas, s/f).

La Comisión Económica para América Latina y el Caribe (Cepal, citada por el Ministerio de Transporte y Obras Públicas, s/f) emitió su ranking anual de puertos 2016, en el cual se analizaron 120 terminales de la región. 
En este estudio la terminal portuaria de Guayaquil ocupa el séptimo lugar con un manejo de 1'821654 TEUS (contenedores de 20 pies), lo que significa un aumento en el manejo de contenedores en comparación al 2015.

\section{Conclusiones}

Los Puertos han sido desde tiempos antiguos centros de comercio estratégicos y representativos para el desarrollo económico de los países. En este sentido, el desarrollo tecnológico y la globalización han contribuido al transporte masivo de mercancías desde largas distancias, lo que sostiene este mecanismo comercial como el más conveniente y rentable convirtiéndose así en la columna vertebral del comercio intercontinental para el transporte a granel de materias primas y la importación y exportación de alimentos y bienes manufacturados.

Colombia se encuentra entre los países latinoamericanos que poseen actividad portuaria comercial privilegiada por su ubicación geográfica. Los puertos de Buenaventura y Cartagena le brindan a Colombia un importante aporte para su desarrollo económico, pues ambos se encuentran dentro ranking de los 20 lugares de los puertos pertenecientes a América Latina y el Caribe, que son publicados por la CEPAL, y que de acuerdo con las cifras del movimiento de contenedores en estos países, dichos puertos ocupan el quinto (5to) y décimo noveno (19no) lugar, representando así un gran logro comercial frente a países fronterizos como un Balboa y Colón, en Panamá; y Santos, en Brasil. 


\section{Referencias Bibliográficas}

ARQHYS.com. (2012). Origen y Evolución de los puertos. Revista ARQHYS, (12). Recuperado de http://www.arqhys.com/construccion/puertos-origen.html.

APM Terminals, (2012). Puerto del callao. Recuperado el 24 de noviembre de 2012, Obtenido de http://www.apmterminals.com/americas/callao/

Autoridad Portuaria de Guayaquil (s/f). El Puerto, obtenido de: http://www.apg.gob.ec/institucional/acerca

Autoridad Portuaria Porto de Santos (s/f). Área de influencia comercial. Obtenido de: http://www.portodesantos.com.br/mercado.php?pagina $=01$

Castro Ruiz, T. (s/f). Sistema Portuario Ecuatoriano. Ministerio de Transporte y Obras Públicas. Recuperado de http://www.sela.org/media/2303887/15-sistema-portuario-ecuatoriano.pdf

CEPAL (2016). Anexos estadísticos EE. Recuperado de http://repositorio.cepal.org/handle/11362/42001

CEPAL. (2014). Boletín marítimo y Logístico 55. Recuperado de: http://www.cepal.org/Transporte/noticias/noticias/5/54565/BoletinMaritimo_55_diciembre2014.pdf

CEPAL (2017). Comercio en contenedores en puertos de la región cae 0,9\% en 2016, Recuperado de https://www.cepal.org/es/comunicados/comercio-contenedores-puerts-la-region-cae-09-2016

CEPAL (2017). Perfil marítimo y logístico de América Latina y el Caribe. Recuperado de https://www.cepal.org/es/infografia/ranking-puertos-top-20-america-latina-caribe-2016

CEPAL (2017). Estudio económico de América latina y el caribe 2016. Recuperado de http://repositorio.cepal.org/bitstream/handle/11362/42001/24/EEE2017_Colombia_es.pdf

CEPAL (2017). Estudio económico de América latina y el caribe 2016. Recuperado de http://repositorio.cepal.org/bitstream/handle/11362/42001/10/EEE2017_Peru_es.pdf

Georgia tech panama logistics innovation and research center (2017). Balboa. Recuperado de: http://logistics.gatech.pa/es/assets/seaports/balboa

Georgia tech panama logistics innovation and research center (2017). Colon Container Terminal. Recuperado de: http://logistics.gatech.pa/es/assets/seaports/colon-container-terminal

González, P. D. (2013). Interempresas (Logistica, Almacenaje y Transporte). Recuperado el 18 de septiembre de 2017, de http://www.interempresas.net/Logistica/Articulos/113831-Eltransporte- internacional.html

Encinas Valenzuela, J. E. (2017). La evolución de los Puertos Marítimos. Instituto de investigaciones estratégicas de la armada de México. Recuperado de http://www.cesnav.edu.mx/ININVESTAM/docs/docs_analisis/da_65-17.pdf

Henao Rendón, G. A. y Henao Rendón, J. A. (2009). Desarrollo y transformación de los puertos en Colombia. Un enfoque desde las competencias laborales. Revista Educación y Humanismo, (17), Recuperado de http://www.unisimonbolivar.edu.co/publicaciones/index.php/educacionyhumanismo 
INEI. (2017). Evolución de exportaciones e importaciones para el año 2017. Recuperado de http://www.inei.gob.pe/media/menurecursivo/boletines/06-informe-tecnico n06_exportaciones-e-importacionesabr2017.pdf

Mincomercio de industria y turismo, (2017). Ley 1 de 1991. Recuperado de http://www.mincit.gov.co/loader.php?IServicio=Documentos\&lFuncion=verPdf\&id=64502\&name=LEY_1_DE _1991.pdf\&prefijo=file

Ministerio de Transporte y Obras Públicas (s/f). Puerto de Guayaquil ocupa el séptimo lugar del top ten de la región. Guayaquil, Ecuador. Noticias. Recuperado de http://www.obraspublicas.gob.ec/puerto-de-guayaquilocupa-el-septimo-lugar-del-top-ten-de-la-region/

Orillac, R. (2006): Conferencia en el foro global de logística y comercio internacional Infraestructura portuaria. Recuperado de: http://www.webpicking.com/notas/orillac.htm

Pérez García, C. (2017). Buenaventura, Cartagena, Santa Marta y Barranquilla, los puertos claves del comercio exterior colombiano. Revista de Logística (05), 22-17. Recuperado de https://revistadelogistica.com/transporte-y-distribucion/buenaventura-cartagena-santa-marta-ybarranquilla-los-puertos-claves-del-comercio-exterior-colombiano/

Rúa Costa, C. (2006) Los puertos en el transporte marítimo. Universidad politécnica de Cataluña. Recuperado de https://upcommons.upc.edu/bitstream/handle/2117/289/8.\%20Rua.pdf 